\title{
Word Sense Disambiguation in Web Content Mining Using Brill's Tagger Technique
}

\author{
S.K.Jayanthi and S. Prema, Member, IACSIT
}

\begin{abstract}
In recent years, great advances have been made in the speed, accuracy, and coverage of automatic word sense disambiguators - systems that given a word appearing in a certain context, can identify the sense of that word. This research has prompted a number of investigations into the relationship between information retrieval (IR) and lexical ambiguity in web mining. The work presented in this paper is such an exploration. Starting with a review of previous research that attempted to improve the representation of documents in IR systems, this research is reassessed in the light of word sense ambiguity. The results of these experiments lead to the conclusions that query size plays an important role in the relationship between ambiguity and IR in web content mining. Word Sense Disambiguation (WSD) is tested and analyzed for some of the existing Information Retrieval engines like Google, Clusty, yahoo, Altavista and msnsearch using Brill's tagger, and the derived results for the IR systems recommends how to accommodate the sense information in the selected document collection. The conclusions of these experiments are found to broadly confirm those of the previous set.
\end{abstract}

Index Terms-Information Retrieval (IR), Query expansion, Vector space model, Visualization, Word Sense Disambiguation (WSD), WordNet, , Web Mining.

\section{INTRODUCTION}

In computational linguistics, word sense disambiguation (WSD) is the process of identifying which sense of a word is used in any given sentence, when the word has a number of distinct senses. WSD is a natural classification problem: Given a word and its possible senses, as defined by a dictionary, classify an occurrence of the word in context into one or more of its sense classes. The features of the context (such as neighboring words) provide the evidence for classification. Sense disambiguation is an "intermediate task" [1] which is not an end in itself, but rather is necessary at one level or another to accomplish most natural language processing tasks. It is obviously essential for language understanding applications such as message understanding, man-machine communication, etc. Developing algorithms to replicate this human ability can often be a difficult task. This research is intended to carry out the issues of WSD in information retrieval systems. Documents related to an Information Retrieval (IR) query sometimes contain only the

Manuscript received October 11, 2010; revised January 17, 2011. This work was supported in part by the Department of Computer Science, K.S.R College of Arts and Science and Research work under Vellar College for Women, Bharathiar University.

S.K.Jayanthi is with Computer Science Department as Associate Professor and Head, Vellalar College for Women (Autonomous), Erode, Tamilnadu, India.

S.Prema is with Computer science Department as Assistant Professor, K.S.R. College of Arts and Science,Tiruchengode-637215, Namakkal district, Tamilnadu, India (e-mail: prema_shanmuga@yahoo.com). synonyms of the query words instead of the query words themselves.

A simple IR system with no knowledge of synonyms fails to recognize the relevance of these documents to the query. So, this research work can improve recall of IR systems by considering the synonyms of the query words as a part of the IR query. However, only relevant synonyms of the query words in the given context contribute useful information to the query. The user can identify these relevant synonyms with the help of Brill's tagging, a disambiguation algorithm. When searching for specific keywords, it is desirable to eliminate occurrences in documents where the word or words are used in an inappropriate sense; for example, when searching for judicial references, it is desirable to eliminate documents containing the word court as associated with royalty, rather than with law ([2], [3], [4]).

\section{StATEMENT OF THE Problem}

Web search engines are the most visible IR applications in Information Retrieval. Nowadays people most often use Google for searching their information in the web using keys. When the user goes for an index based search, the key may have disambiguation such as synonymy, polynymy, hepernymy, hyponymy and metonymy. This research focuses on those disambiguation problems raised in current information retrieval search engines like Google, Cluster, yahoo, Altavista and msnsearch. One problem with word sense disambiguation is deciding what the senses are. In cases like the word bass above, at least some senses are obviously different. In other cases, however, the different senses can be closely related (one meaning being a metaphorical or metonymic extension of another), and in such cases division of words into senses becomes much more difficult. Different dictionaries will provide different divisions of words into senses. One solution some researchers have used is to choose a particular dictionary, and just use its set of senses. Generally, however, research results using broad distinctions in senses have been much better than those using narrow, so most researchers ignore the fine-grained distinctions in their work. Another problem is inter-judge variance. WSD systems are normally tested by having their results on a task compared against those of a human. However, humans do not agree on the task at handgive a list of senses and sentences, and humans will not always agree on which word belongs in which sense. A computer cannot be expected to give better performance on such a task than a human (indeed, since the human serves as the standard, the computer being better than the human is incoherent), so the human performance serves as an upper bound. Human performance, however, is much better on 
coarse-grained than fine-grained distinctions, so this again is why research on coarse-grained distinctions is most useful. Some AI researchers like Douglas Lenat argue that one cannot parse meanings from words without some form of common sense ontology. To properly identify senses of words one must know common sense facts [5],[6]. The inherent difficulty of sense disambiguation was a central point in Bar-Hillel's well known treatise on machine translation [7]), where he asserted that he saw no means by which the sense of the word pen in the sentence. The box is in the pen could be determined automatically.

\section{Word SENSE AMBIGUITY IN IR}

Information retrieval (IR) is the science of searching for documents, for information within documents and for metadata about documents, as well as that of searching relational databases and the World Wide Web. IR is a component of an information system. An information system must make sure that everybody it is meant to serve has the information needed to accomplish tasks, solve problems, and make decisions, no matter where that information is available. To this end, an information system must (1) aggressively find out what users need, and acquire documents (or computer programs, or products, or data items, and so on), resulting in a collection, and (3) match documents with needs.

Automated information retrieval systems are used to reduce what has been called "information overload". Many universities and public libraries use IR systems to provide access to books, journals and other documents. Web search engines are the most visible IR applications. An IR system is affected by the characteristics of text, one such characteristics word sense ambiguity. Most words are ambiguous to some degree, what sense a word occurrence has depends on the context it appears in. For some words, their senses are unrelated, for example the word 'window' could refer to an implement used in graphics or for interior design .For most words however, and their senses are related. As IR systems process written text, they are affected by word sense ambiguity. An example of such an effect was reported in a personal communication with the author. A manager of an on-line news retrieval system found queries about the current CEO of XY concern were causing problems with their IR system. A number of users had tried to retrieve articles about the CEO using the query 'Executive'. Wilks tested the accuracy of his disambiguator on the word 'bank' as it appeared in around 200 sentences [1]. This query caused many articles about 'John Major' to be retrieved, but in addition many more articles were retrieved where 'major' was used as an adjective or as the name of a military rank. Word sense ambiguity is not something encountered by people in every day life, except perhaps in the context of jokes. Somehow, when an ambiguous word is used in a sentence, people are usually able to select the correct sense of that word without conscious effort. This manual word sense disambiguating (WSD) ability has been investigated, an overview of which can be found in, working with the French language, found that people could accurately determine the sense of a particular word from reading the previous two words alone. Miller briefly describes similar work by Kaplan using the English language which seems to draw similar results to those of Choueka and Lusignan. These works show that accurate disambiguation can be performed without exposure to the wider context in which an ambiguous word appears. Automatic WSD systems have been studied for many years - Gale, Church, and Yarowsky [8] cite work dating back to 1950 . For many years disambiguators could only accurately disambiguate the text of tightly focused subject areas. In recent years this situation has changed and the accuracy, speed, and scalability of disambiguators have improved. This improvement is such that it is now possible to apply a disambiguator to a document collection of wide ranging texts and expect it to disambiguate the text accurately. Retrieval can be performed on such a collection using an IR system, and because the words of that collection are represented as word senses, the quality of document retrieval can reasonably be expected to improve. It is an investigation of this possibility that forms the basis of this thesis. Despite the increasing importance of Information Retrieval (IR) systems as data retrieval tools, the performance of most of these systems has not yet reached a satisfactory level. Word sense ambiguity is one of the reasons for their poor performance. Overcoming this problem may improve IR performance. This research brings together the WSD issues in information retrieval and proposes a method for solving this problem in web content mining.

\section{The Proposed Methodology}

\section{A. The Current NLP Approaches for WSD}

As in all natural language processing, there are two main approaches to WSD - deep approaches and shallow approaches. Deep approaches presume access to a comprehensive body of world knowledge. Knowledge, such as "you can go fishing for a type of fish, but not for low frequency sounds" and "songs have low frequency sounds as parts, but not types of fish", is then used to determine in which sense the word is used. These approaches are not very successful in practice, mainly because such a body of knowledge does not exist in a computer-readable format, outside of very limited domains. However, if such knowledge did exist, then deep approaches would be much more accurate than the shallow approaches. Also, there is a long tradition in computational linguistics, of trying such approaches in terms of coded knowledge and in some cases; it is hard to say clearly whether the knowledge involved is linguistic or world knowledge. The first attempt was that by Margaret Masterman and her colleagues, at the Cambridge Language Research Unit in England, in the 1950s. This attempt used as data a punched-card version of Roget's Thesaurus and its numbered "heads", as an indicator of topics and looked for repetitions in text, using a set intersection algorithm. It was not very successful, as is described in some detail in [1], but had strong relationships to later work, especially Yarowsky's machine learning optimization of a thesaurus method in the 1990s. Shallow approaches don't try to understand the text. Word sense ambiguity is a pervasive characteristic of natural language. For example, the word "cold" has several senses and may refer to a disease, a 
temperature sensation, or a natural phenomenon. The specific sense intended is determined by the textual context in which an instance of the ambiguous word appears. In "I am taking aspirin for my cold" the disease sense is intended, while in "Let's go inside, I'm cold" the temperature sensation sense is meant. These rules can be automatically derived by the computer, using a training corpus of words tagged with their word senses. This approach, while theoretically not as powerful as deep approaches, gives superior results in practice, due to the computer's limited world knowledge. However, it can be confused by sentences, like the dogs bark at the tree, which contains the word bark near both tree and dogs. These approaches normally work by defining a window of $N$ content words around each word to be disambiguated in the corpus, and statistically analyzing those $N$ surrounding words. Two shallow approaches used to train and then disambiguate are Nä̈ve Bayes classifiers and decision trees. In recent research, kernel based methods such as support vector machines have shown superior performance in supervised learning. But over the last few years, there hasn't been any major improvement in performance of any of these methods.

\section{B. A Study on WSD Algorithms}

It is instructive to compare the word sense disambiguation problem with the problem of part-of-speech tagging. Both involve disambiguating or tagging with words, be it with senses or parts of speech. However, algorithms used for one do not tend to work well for the other, mainly because the part of speech of a word is primarily determined by the immediately adjacent one to three words, whereas the sense of a word may be determined by words further away. The success rate for part-of-speech tagging algorithms is at present much higher than that for WSD, state-of-the art being around $95 \%$ accuracy or better, as compared to less than $75 \%$ accuracy in word sense disambiguation with supervised learning. These figures are typical for English, and may be very different from those for other languages. Another aspect of word sense disambiguation that differentiates it from part-of-speech tagging is the availability of training data. While it is relatively easy to assign parts of speech to text, training people to tag senses is far more difficult. While users can memorize all of the possible parts of speech a word can take, it is impossible for individuals to memorize all of the senses a word can take. Thus, many word sense disambiguation algorithms use semi-supervised learning, which allows both labeled and unlabeled data. The Yarowsky algorithm was an early example of such an algorithm. Yarowsky's unsupervised algorithm uses the 'One sense per collocation' and the 'One sense per discourse' properties of human languages for word sense disambiguation. From observation, words tend to exhibit only one sense in most given discourse and in a given collocation. The corpus is initially untagged. The algorithm starts with a large corpus, in which it identifies examples of the given polysemous word, and stores all the relevant sentences as lines. For instance, Yarowsky uses the word 'plant' in his 1995 paper to demonstrate the algorithm. The residual examples (85\% $98 \%$ according to Yarowsky) remain untagged. According to the criteria given in Yarowsky [8], seed words that appear in the most reliable collocational relationships with the target word will be selected. The effect is much stronger for words in a predicate-argument relationship than for arbitrary associations at the same distance to the target word, and is much stronger for collocations with content words than with function words.

\section{The Proposed Method Using Brill Tagger}

The Brill tagger is a method for doing part-of-speech tagging. It was described by Eric Brill in his $1993 \mathrm{PhD}$ thesis [9]. It can be summarized as an error-driven transformation-based tagger. It is error-driven in the sense that it recourses to supervised learning. Transformation-based in the sense that a tag is assigned to each word and changed using a set of predefined rules. Note: If the word is known, it first assigns the most frequent tag, or if the word is unknown, it naively assigns the tag "noun" to it. Applying over and over these rules, changing the incorrect tags, a quite high accuracy is achieved. The algorithm goes as follows:

Initialization:

- Known words (in vocabulary): assigning the most frequent tag associated to a form of the word

- Unknown words (out of vocabulary) :

○ Proper noun if capitalized and simple noun else

- Learning or guessing rules on the same basis as contextual rules

Learning Phase:

- Iteratively compute the error score of each candidate rule (difference between the number of errors before and after applying the rule)

- Select the best (higher score) rule.

- Add it to the rule set and apply it to the text.

Repeat until no rule has a score above a given threshold (that is, until applying new rules leaves the text in the same state, which is then supposed to be the final state of the tagging).

The figure below shows some of the selectors of the word "charge" in two different sentences. The examples show that contexts play an important role in finding selectors which enable the user to identify the correct sense of an ambiguous word. The proposed IR architecture [12] has been given in Figure 1.In existing search engines the result is in URL mode whereas in the proposed work the clustered result [11] is in visual mode.

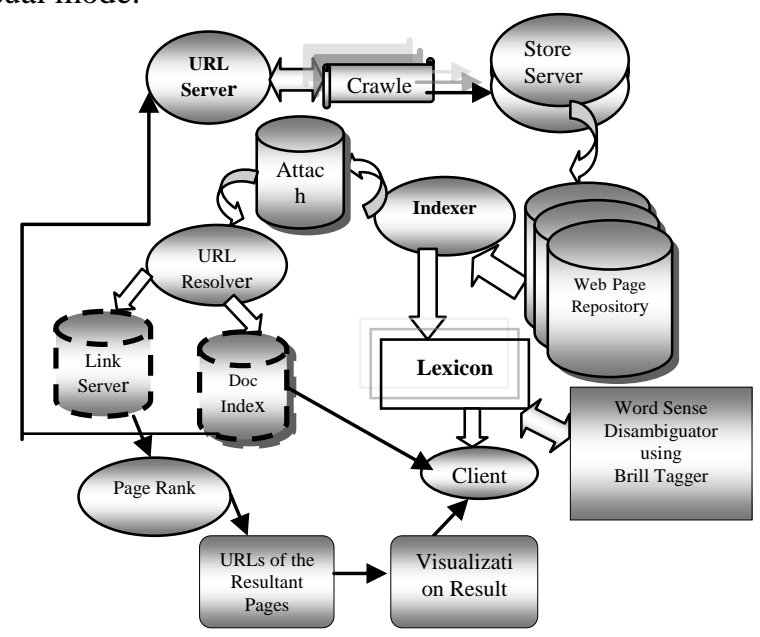

Figure 1. The Proposed IR Architecture using Brill Tagger 


\section{RELATED WORKS}

There exist many techniques that are used for word sense disambiguation. Schutze labels this task as "word sense discrimination". He distinguishes this from "sense labeling" where each sense receives the correct label. This distinction often — but not always — coincides with unsupervised (discrimination) versus supervised (labeling) machine learning techniques [4]. Unsupervised learning algorithms learn patterns solely from input parameters without trying to match to pre-specified categories. For example, Pedersen and Bruce tested three unsupervised learning algorithms: Wards and McQuitty's clustering and the EM algorithm. They mapped these clusters to dictionary senses so that there was maximal agreement. Supervised learning is used more often for WSD. The supervised learning techniques rely on a training set comprised of example ambiguous words and their correct sense. Decision trees, such as ID3 or C4.5, artificial neural networks (ANN), such as the feed forward/ back propagation ANN, and probabilistic based methods, such as Nave Bayes, are commonly used. Mooney tested seven such supervised learning methods with the word line. His work demonstrates the importance of a large dataset. In addition to different algorithms, the amount, relevance, and precision of the information affect performance. Hoste et al. manipulated the feature information and algorithm settings in an extensive set of studies. Pedersen evaluated the use of bigrams (sequences of two words) for WSD with a decision tree and Navve Bayes classifier. Florian et al. worked with the Senseval-2dataset (www.itri.brighton.ac.uk/events/senseval/) . In later studies, they combined different types of classifiers, such as vector-based methods (e.g., naive Bayes), variance-based methods (e.g., Maximum Variance Correction), and Brill's transformation-based learning. They also evaluated different feature spaces such as words, lemmas, and part-of-speech tags in different settings such as traditional bag-of-word approaches, but also local bigram, trigram, and other syntactic relationships. For example, Mihalcea and Moldovan based their approach on semantic density between words and focus on verb-noun pairs [10]. Their distance measure is based on an evaluation of common words between two sets of words. They use WordNet as their knowledge source and calculate statistics for the most probable senses using the Internet as a corpus. Hoste et al. describe a memory-based learning approach where the algorithm keeps all training examples in memory. A classification decision is made based on similarity between new input and stored examples. MetaMap, provided by the National Library of Medicine, uses rules to map between words in the text and UMLS Met thesaurus and provides a score to indicate the fit of each mapping.

\section{RESULTS}

The results of both sets of experiments presented in this paper proved to be complementary. The main contributions derived from them are as follows. Query size has been shown to play an important role in determining the impact of ambiguity on retrieval effectiveness in web content mining [13]. Retrievals based on queries composed of one or two words were considerably affected by ambiguity, those based on longer queries were much less affected. The errors made by disambiguators were found to have a significant impact on effectiveness, so much so, that disambiguation was only worth performing when the accuracy of the disambiguator was high. The only time that disambiguation was found to provide any utility to retrieval effectiveness was for retrievals based on short queries [14]. The analysis of the frequency distribution of word senses provides an explanation for these results. This analysis in Fig.2 shows that the skewed distribution of the senses of a word caused ambiguity to be not as significant to retrieval effectiveness as might have been thought.

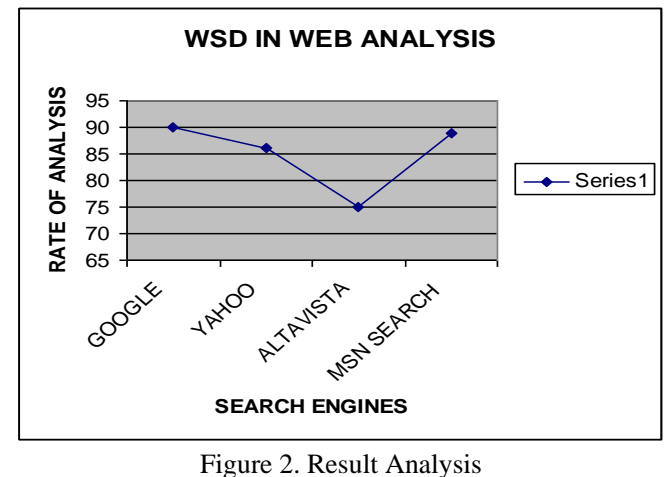

\section{CONCLUSION}

Word sense ambiguity is recognized as having a detrimental effect on the precision of information retrieval systems in general and web search systems in particular, due to the sparse nature of the queries involved. Despite continued research into the application of automated word sense disambiguation, the question remains as to whether less than $90 \%$ accurate automated word sense disambiguation can lead to improvements in retrieval effectiveness. This research paper explored the development and subsequent evaluation of word sense disambiguation using Brill tagger for several search engines which demonstrates increased precision from a sense based vector space retrieval model over traditional TF*IDF techniques. In this paper, the effectiveness of an IR system retrieving from a disambiguated collection was measured. The main result of these experiments is the drop in retrieval effectiveness resulting from the use of the sense information produced by the disambiguator. As already been stated, it is believed that the relatively poor accuracy of the disambiguator is to blame for this and this paper contains some suggestions on how this might be improved. Despite the errors in its output, use of the disambiguator's sense information was found to improve retrieval effectiveness for very short queries and this improvement is believed to be the first demonstration of disambiguation (based on a dictionary or thesaurus) being beneficial to retrieval effectiveness. Another aspect of the experiments was the comparison of two methods for accommodating sense information in an IR system: full-sense/variation distance; and single-sense/binary matching. As only a single case of using disambiguation information was shown to be beneficial to retrieval effectiveness, no conclusions can be made on the merits of 
one method over another. The result of this one case, however, showed that use of the full-sense/variation distance method produced better effectiveness than use of the other. This is countered slightly by the result showing the single-sense/binary-matching method marginally improving effectiveness measured at low recall. Although taken on their own, these results are disappointing, in the wider context of the other ambiguity experiment of the thesis, the results are complementary. Ambiguity is not as significant a problem to effectiveness as might have been thought, except for retrievals based on short queries. Disambiguation is only useful to an IR system if the disambiguator is accurate. The benefits of a disambiguator are greatest for retrievals based on short queries.

\section{REFERENCES}

[1] Y.Wilks and M. Stevenson," The grammar of sense: is word-sense tagging much more than part-of-speech tagging" Technical report, University of Sheffield, UK.1996.

[2] G.Salton,"Automatic Information Organization and Retrieval", Ed. New York: McGraw-Hill, 1968.

[3] G. Salton and M.McGill,'Introduction to Modern Information Retrieval", Ed. New York: McGraw-Hill,1983.

[4] H. Schütze, and J. Pederson,'Information Retrieval based on word senses. Symposium on Document Analysis andInformation Retrieval (SDAIR). Las Vegas, Nevada, 1995, 161-175.

[5] Y.Wilk," Close Engagements with Artificial Companions: Key Social, Psychological and Design issues". John Benjamins, Amsterdam, 2010.

[6] L.Guthrie,J. Pustejovsky,B.Slator and Y.Wilks," The Role of Lexicons in Natural Language Processing.Communications of the ACM", Special Issue on Natural Language Processing, January 1996, Vol. 39 No. 1, pp. 63-72.

[7] Y.Bar-Hillel, "Some reflections on the present outlook for high-quality machine translation". Feasibility study on fully automatic high quality translation, ed. Winfred P.Lehmann and Rolf Stachowitz, 4pp. Griffiss Air Force Base, New York: Rome AirDevelopment Center, Air Force Systems Command. (RADC-TR-71-295),1999

[8] David Yarowsky, X.Y.Chou, "Yarowsky's unsupervised algorithm", Oxford Computing Lab, 2007.

[9] The Brill tagger is a method for doing part-of-speech tagging. It was described by Eric Brill in his 1993 PhD thesis

[10] Mihalcea and Moldovan, RANLPIR '00 Proceedings of the ACL-2000 workshop on Recent advances in natural language processing and information retrieval: held in conjunction with the 38th Annual Meeting of the Association for Computational Linguistics - Volume 11. 2003.
[11] S.Sasikala and Dr.S.K.Jayanthi,"Link Spam Detection based on DBSpamClust with Fuzzy C-Means Clustering”, International Journal of Next-Generation Networks (IJNGN), Vol IV Dec 2010,pp.1-8.

[12] S.Prema and Dr.S.K.Jayanthi,'Facilitating Efficient Integrated Semantic Web Search with Visualization and Data Mining Techniques", International Conference on Information \& Communication Technologies, Springer Transl.sep, 2010,pp. 437 442.

[13] S.Prema and Dr.S.K.Jayanthi,'Bookshelf Data Structure Incorporated Visualization for Efficient Integrated Semantic Web Search", International Conference On Emerging Trends In Mathematics And Computer Applications - 2010, Proc. Mepco shlenk college of engineering ,Dec. 2010

[14] Vijaya kathiravanan," E-mail id harvester to retrieve E-mail addresses of domain experts", In: National Conference On Current Trends in Computer Applications, 2009.

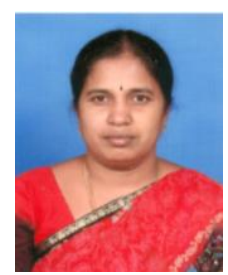

Dr.S.K.Jayanthi received the M.Sc., M.Phil., PGDCA, Ph.D in Computer Science from Bharathiar University in 1987, 1988, 1996 and 2007 respectively. She is currently working as an Assosiate Professor, Head of the Department of Computer Science in Vellalar College for Women. She secured District First Rank in SSLC under Backward Community. Her research interest includes Image Processing, Pattern Recognition and Fuzzy Systems. She has guided 18 M.Phil Scholars and currently 4 M.Phil Scholars and 4 Ph.D Scholars are pursuing their degree under her supervision. She is a member of ISTE, IEEE and Life Member of Indian Science Congress. She has published 5 papers in International Journals and one paper in National Journal and published an article in Reputed Book. She has presented 14 papers in International level Conferences/Seminars, 16 papers in National level Conferences/Seminars and participated in around 35 Workshops/Seminars/Conferences/FDP.

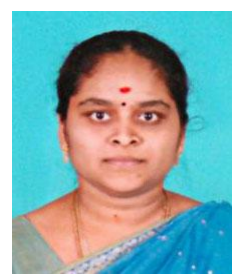

S.Prema, currently working as a Assistant Professor in K.S.R. College of Arts \& Science has received the B.Sc., M.C.A., M.Phil., from the Periyar University in 2001,2004,2008 respectively and now pursuing Ph.D in computer science at Bharathiar University. Her area of Doctoral research is Web mining. She has been a First rank holder under Periyar University in B.Sc Programme. She has published one paper in international journal of Advances in Computational Sciences and Technology (ACST). She has presented 10 papers in International Conferences/Seminars, 18 papers in National Conferences/Seminars and participated in 4 National Conferences/Seminars, 7 workshops and 1 FDP. 